\title{
High Case Volumes and Surgical Fellowships are Associated with Improved Outcomes for Bariatric Surgery Patients: A Justification of Current Credentialing Initiatives for Practice and Training
}

\author{
Geoffrey P Kohn, MBBS (Hons), FRACS, Joseph A Galanko, PhD, D Wayne Overby, MD, and \\ Timothy M Farrell, MD, FACS \\ Department of Surgery, University of North Carolina at Chapel Hill, NC
}

\begin{abstract}
Background-Recent years have seen the establishment of bariatric surgery credentialing processes, centers-of-excellence programs and fellowship training positions. The effects of centerof-excellence status and of the presence of training programs have not previously been examined. The objective of this study is to examine the effects of case volume, center-of-excellence status and training programs on early outcomes of bariatric surgery.
\end{abstract}

Study Design-Data were obtained from the Nationwide Inpatient Sample from 1998 to 2006. Quantification of patients' comorbidities was made using the Charlson Index. Using logistic regression modeling, annual case volumes were analyzed for an association with each institution's center-of-excellence status and training program status. Risk-adjusted outcome measures were calculated for these hospital-level parameters.

\begin{abstract}
Results-Data from 102,069 bariatric operations were obtained. Adjusting for comorbidities, greater bariatric case volume was associated with improvements in the incidence of total complications (odds ratio $[\mathrm{OR}]=0.99937$ for each single case increase, $\mathrm{p}=0.01$ ), in-hospital mortality $(\mathrm{OR}=0.99717, \mathrm{p}<0.01)$, and most other complications. Hospitals with a Fellowship Councilaffiliated gastrointestinal surgery training program were associated with risk-adjusted improvements in rates of splenectomy $(\mathrm{OR}=0.2853, \mathrm{p}<0.001)$ and bacterial pneumonias $(\mathrm{OR}=0.65898, \mathrm{p}=0.02)$. Center-of-excellence status, irrespective of the accrediting entity, had minimal independent association with outcome. A surgical residency program had a varying association with outcomes.

Conclusions-The hypothesized positive volume-outcome relationship of bariatric surgery is shown without arbitrarily categorizing hospitals to case volume groups, by analysis of volume as a continuous variable. Institutions with a dedicated fellowship training program have also been shown, in part, to be associated with improved outcomes. The concept of volume-dependent center-ofexcellence programs is supported, though no independent association with the credentialing process is noted.
\end{abstract}

(C) 2010 American College of Surgeons. Published by Elsevier Inc. All rights reserved.

Correspondence address: Dr Geoffrey P. Kohn, University of Southern California, Department of Surgery, 1510 San Pablo St, Suite 514, Los Angeles, CA 90033, Tel (323)442-9066, Fax (323)442-5872, gkohn@ UpperGI.net.

Publisher's Disclaimer: This is a PDF file of an unedited manuscript that has been accepted for publication. As a service to our customers we are providing this early version of the manuscript. The manuscript will undergo copyediting, typesetting, and review of the resulting proof before it is published in its final citable form. Please note that during the production process errors may be discovered which could affect the content, and all legal disclaimers that apply to the journal pertain.

Disclosure Information: Nothing to disclose.

This paper was presented at the Residents and Fellows session of the 2009 Annual Meeting of the Society of American Gastrointestinal and Endoscopic Surgeons, Phoenix, AZ, where it was awarded the Best Paper Prize. 


\section{Introduction}

The United States has experienced a steady rise in the prevalence of obesity over the last 20 years ${ }^{1}$. At the turn of the millennium, nearly two-thirds of Americans were overweight or obese, and almost $5 \%$ were morbidly obese ${ }^{2}$. Obesity shortens life expectancy and is projected to soon overtake smoking as the leading cause of death in the United States ${ }^{3}$.

Many surgeons are now pursuing advanced training in bariatric surgery. Professional organizations such as the American Society for Metabolic \& Bariatric Surgery (ASMBS) and the Fellowship Council have developed curricular guidelines and program accreditation algorithms to define acceptable fellowship training 1, 4, 5. As well, the ASMBS and the American College of Surgeons (ACS) have established Center-of-Excellence standards and certification programs in an effort to direct case flow to the best practices. Insurance and malpractice providers have quickly come to embrace these guidelines and certification programs.

There exist limited data in the literature driving these initiatives. There is some support for a relationship between higher bariatric case volumes and lower complications, but very little evidence for an independent impact of Center-of-Excellence status, fellowship training or an accredited surgical residency program.

\section{Methods}

The most recently available Nationwide Inpatient Sample (NIS) 2 databases covering the years 1998-2006 were queried. These are the largest all-payer inpatient care databases in the United States, containing data from approximately 8 million hospital stays each year. The latest release, the 2006 database, contains all discharge data from 1,045 hospitals located in 38 States, approximating a 20-percent stratified sample of all non-Federal, short-term, general, and other specialty hospitals in the United States 2 . The stratification means that non-included states are thought to have little impact on subsequent analyses 6 . The stratification process is complex but well-validated, and strata are defined based on five hospital characteristics: geographic region, control (for example, governmental or private), location (urban or rural), teaching status and bed size 6 . A dataset was created by merging core and hospital files, and filtered according to specific criteria previously published 7,8 (Table 1 ). Various search strategies exist to capture all bariatric operations; the strategy utilized here is one of the more inclusive ${ }^{9}$. To estimate nationwide case volume totals the discharge-level weight was applied. At all other times, the unweighted NIS cohort was utilized, without application of weights; this was particularly important when calculating standard errors and performing regression analyses. The benefit of performing the analysis on the unweighted sample is that the results are calculated from observed data, rather than estimated from the nationwide sample and without the introduction of sampling errors of infrequent complications based on imprecise stratification. The downside of this approach is the inability to generalize the results for the United States population as a whole. Bariatric surgery patients whose stay in hospital was $\leq 23$ hours - predominantly those undergoing laparoscopic adjustable gastric band procedures - were not captured by the NIS database.

The presumed operation was based on the recorded ICD-9-CM procedure code, with the groups described in Table 2.

Information regarding the presence of a fellowship program in each year of the study period was taken from the Fellowship Council's webpage ${ }^{3}$.

Details of Center-of-Excellence (COE) status were obtained from the websites of the American College of Surgeons ${ }^{4}$ and the Surgical Review Corporation (following the link from the 
American Society for Metabolic and Bariatric Surgery website) 5. Only hospitals currently designated as $\mathrm{COE}$ could be identified from these sites - it was assumed that current designation as such was in place throughout the study period. For reasons explained in the discussion, another method of determination of COE status was obtained by querying the list of Medicareapproved centers-of-excellence for the year 20068 . The NIS hospital universe was searched for all COE hospitals obtained from the aforementioned websites. NIS hospital identification numbers were determined for all hospitals included in both groups, and appropriate notation was added to the discharge level entry in the NIS dataset.

A teaching hospital is defined by the NIS as being a hospital with residents in any specialty. Hospitals defined as having a surgical residency were defined as a subgroup. Details of such a surgical residency program were obtained by combining information from the American Medical Association's FREIDA database and the listings of accredited programs on the Accreditation Council for Graduate Medical Education webpage ${ }^{6,7}$.

Comorbidity scores were applied to each inpatient stay record, using the Deyo adaptation of the Charlson comorbidity index ${ }^{10}$. This validated index allocates a score between 0 and 35 , with a higher score indicating more comorbidity. The comorbidities examined include: one point for each of myocardial infarction, congestive heart failure, peripheral vascular disease, cerebrovascular disease, dementia, pulmonary disease, connective tissue disease, peptic ulcers and mild chronic liver disease, diabetes; two points for each of hemiplegia, renal disease, diabetes-related complications, malignancy and leukemia; three points for moderate-severe chronic liver disease; and six points for metastatic cancer and acquired immune deficiency syndrome.

Perioperative complications were added based on ICD-9-CM code, in a similar manner to that described by Santry ${ }^{7}$. The diagnosis of "any complication" was made if the in-hospital death field was true, or if any of the NIS's 15 diagnosis codes included: abdominal drainage procedures (5491), acute cerebrovascular accident (43100-43191, 4330-4339, 4340-43491), acute dialysis (3895), acute deep venous thrombosis $(4538,4539)$, acute myocardial infarction (4100-4109), acute pulmonary embolism $(4151,41511,41519)$, acute renal failure (58415849), acute respiratory failure (51881), adhesiolysis (5451, 5459), anastomotic leak (9986), bacterial pneumonia (481, 485, 486, 4820-4829), cardiac complications (9971), central nervous system complications (99701-99703), dialysis catheter insertion (3995), foreign body removal (5492), intraoperative hemorrhage (99811), laparotomy (5412), mechanical ventilation $(967,9671,9672,9673)$, postoperative shock $(9980)$, reclosure of abdomen (5461), respiratory tract complications (99973), small bowel obstruction (5600-5609), splenectomy (4143, 415), splenic injury (8650-8651), tracheostomy (311, 3129), transfusion (9904, 9909), urinary complications (9975), wound dehiscence $(9983,99831,99832)$, wound infection $(9985$, 99851, 99859), wound seroma (99813) and death.

\section{Statistics}

SAS 9.2 (SAS Institute, Cary, NC) was used to analyze the data. Logistic regression modeling was performed using generalized estimating equations and assuming a binomial distribution of the data. This allowed certain covariables to be controlled for; unless otherwise specified, these included annual improvements in outcomes and Charlson comorbidity index scores. Controlling for individual bariatric procedures was not performed, but for reasons detailed below, outcomes were analyzed for all bariatric procedures and for all non-adjustable gastric band bariatric procedures. Repeated measures analysis was performed with the experimental unit being hospital identification number clusters. We used logistic regression modeling to model the dichotomous response variables. We used compound symmetric correlation to account for expected correlation within individual hospitals. We used reference cell coding for our parameterization. The model was fit and empirical standard error estimates were generated 
which in turn were used to generate p-values. A $p$-value $<0.05$ was considered significant. Subsequently, the estimates were exponentiated to calculate an odds ratio and $95 \%$ confidence intervals.

We fit the models first with the quadratic terms, for which $p$-values were generated. If statistical significance was determined, then the quadratic term was retained in the model. Otherwise, the quadratic was removed and a linear model was utilized. In interest of simplicity of presentation of our data, tables were presented using the linear model, while graphical representation of the case volume / outcome relationship allowed for demonstration of the model with the quadratic terms, which had reached significance.

\section{Results}

A cohort of 102,069 bariatric cases was identified from the database for the study period. Applying the appropriate NIS weights, this estimates a nationwide total of 496,267 inpatient bariatric cases were performed in the United States during the study period of 1998-2006. Nearly two-thirds of bariatric operations were performed in teaching hospitals, a proportion which stayed stable over the study period (Table 3 ). The majority of operations were performed in the largest third of hospitals.

Case volume requirements have been determined by the two Center-of-Excellence (COE) credentialing bodies and differ by accreditation category. For ASMBS accreditation, institutions are required to perform either 125 or 100 bariatric operations per year, depending on whether the practice is hospital-based or free-standing. The ACS has defined various levels of accreditation, with cases volume requirements ranging from 125 per year to 50 per year.

As shown in Table 4, a relatively stable number of bariatric programs are meeting the volume requirement for top level COE accreditation.

Concurrent with an increase in hospitals achieving greater case volumes, the in-hospital mortality rate for bariatric operations taken as a whole has steadily decreased throughout the study period, despite an increasing comorbidity score of the patients over the same time frame (Table 5). Overall mortality rates for all bariatric procedures were $0.61 \%$ in $1998,0.23 \%$ in 2002 and $0.13 \%$ in 2006. In-hospital mortality rates of laparoscopic adjustable gastric bands were consistently less than those of gastric bypasses, which were in turn consistently less than malabsorptive operations such as biliopancreatic diversion.

To determine the effect on complications of case volume, center-of-excellence status, and training programs, a generalized linear model was applied as described above. Regarding operations performed with such a large frequency, any decrease in complication rate was thought to be clinically significant, as the large numbers would greatly multiply any effect. Firstly, the entire bariatric procedures cohort was modeled. With an increasing number of laparoscopic adjustable gastric banding procedures occurring late in the study period in an ambulatory setting ${ }^{9}$, many of these operations were not captured in the Nationwide Inpatient Sample. Therefore, modeling was also performed on the total of non-band bariatric operations.

Table 6 examines the independent association of annual hospital case volume with complication rates, after controlling for year (improvements in outcomes may have occurred over the study period) and for Charlson comorbidity scores. In contrast to previous studies, artificial case volume groups were not applied and analysis was performed with case volume as a continuous variable. An odds ratio $<1.0$ signifies an inverse association of case volume with the complication under review. Nearly all analyzed complication categories showed an inverse correlation with case volume, with any complication, death, requirement for postoperative abdominal drainage, acute $\mathrm{PE}$, cardiac complications, splenectomy, acute renal 
failure, bacterial pneumonia and acute respiratory failure rates achieving statistically significant improvement. The odds ratios tend to be very close to 1.0 because the ratios represent the effect of increasing the annual volume by a single case.

Figure 1 uses data modeled from the year 2006 to graph the predicted association of case volume with mortality by Charlson comorbidity score.

Table 7 describes the independent association of the presence of a Fellowship Counciladministered Gastrointestinal Surgery fellowship program, after controlling for year, Charlson comorbidity scores and case volume. A fellowship was associated with fewer splenectomies and bacterial pneumonias, but more DVTs.

Table 8 describes the independent association of the presence of an ACGME-accredited surgical residency program, after controlling for year, Charlson comorbidity scores and case volume. A significant increase in the rate of cardiac complications and myocardial infarction was observed, as well as of acute pulmonary embolism. Splenectomy rates and rates of respiratory complications (bacterial pneumonia and acute respiratory failure) were inversely correlated.

Table 9 describes the independent association of the presence of Center-of-Excellence designation according to the ASMBS and the ACS after controlling for year, Charlson comorbidity score and case volume. There was a significant independent association of respiratory failure alone with an ASMBS designation of center-of-excellence.

An ACS designation of center-of-excellence was associated with a significant decrease in the incidence of postoperative respiratory failure as well the rate of any complication when only non-gastric band patients were considered.

Table 10 narrows the search of COE centers to those recognized by Medicare in the year 2006. Acute renal failure and postoperative shock state were significantly improved in this year in facilities which had met Medicare's minimum standards for bariatric surgery.

\section{Discussion}

As the rate of morbid obesity in the US increases, the number of patients who would benefit from bariatric surgery continues to grow. The increasing number of training programs dedicated to bariatric surgery and the increasing number of institutions performing these procedures reflect the importance given to this problem by the medical community.

The management of the bariatric patient is often quite complex so realizing the best possible outcomes for these patients may require changes to standard institutional facilities, and to the practices of staff and physicians. Institutions must have furniture, hospital equipment and imaging suites suitable for high body mass index patients. Staff members need to be competent and compassionate when dealing with the medical and psychological complications of obesity. Surgeons must have an understanding of the perioperative and long-term risks of bariatric surgery, and be experienced in the operative care of the morbidly obese ${ }^{5,11}$.

A strong association between case volume and outcomes has been demonstrated for many surgical procedures. Bariatric surgery was expected to demonstrate a similar relationship, and early studies suggested that this might be true ${ }^{12-14}$. Most early reports divided centers into arbitrarily determined case volume categories, frequently using an annual case volume of 100 as the distinction between low-volume and high-volume centers, and examined very few complications. Nonetheless, these findings soon led to widespread belief in the necessity of ensuring bariatric cases were directed to high-volume centers. Soon thereafter, center-of- 
excellence accreditation programs were devised, based on these arbitrary thresholds, and this development was quickly linked to remuneration by third-party payers. However, there has been very little published validation of this paradigm, and post-COE-introduction outcomes have not been reported.

The vast number of cases captured in the NIS dataset allows very small differences between groups to be detected. With over 100,000 bariatric cases available for review, low frequency outcomes such as death, pulmonary embolism and others can be examined. The numbers also allow modeling with continuous variables rather than with the arbitrary assignment of categorical groups. The use of the NIS dataset does entail some limitations, one of the most important of which is the absence of a longitudinal identification number for patients. Thus, once a patient is discharged from hospital, that patient is lost to follow-up through the database; any re-admission will not be captured.

The most striking finding of our analysis is the association of case volume with outcomes. The magnitude of the positive association of volume with outcomes was small and required the large numbers of the NIS dataset for detection, but was evident for total complications, death, requirement for postoperative percutaneous abdominal drainage, acute pulmonary embolus, cardiac complications and postoperative in-hospital myocardial infarction, incidental splenectomy, post-operative physiological shock states, renal failure, postoperative respiratory failure and bacterial pneumonia. These improvements in outcomes increased with increasing case volume. However, and very importantly, it appears that the positive association of many outcomes with volume, including the outcome of mortality rates, improves continuously with ever-increasing case volume. While it may be reasonable to establish case volume thresholds tied to "acceptable" complication rates for credentialing purposes, the authors emphasize that complications continue to improve at still higher case volumes. These improvements, though statistically significant, are nonetheless small in magnitude.

It might be argued that the study design, encompassing many years, might have some limitations, with the recent increases in annual case volumes being reflected in increasing experience, and the early years not being representative of the current environment. This argument is countered by the inclusion of year as a covariable in the analysis in a speculative attempt to negate this time-related effect. Additionally, it might be argued that any riskstratification not including BMI as a predictor might limit accurate prediction of complications. However, since BMI is uncommonly reported by coders to the NIS, we determined it could not usefully be added to the risk stratification model. Irrespective, the use of the Charlson Comorbidity Score is a multiply-validated technique of risk stratification of the surgical patient, and in spite of not including BMI as a predictor, is thought to be a valid and robust prognostic index to use.

It is interesting to note that the independent association with outcomes of both ACS and ASMBS designated Centers of Excellence was quite small, a finding supported by a previous study by Livingston ${ }^{15}$. Even though lower rates for total complications and deaths were measured in Centers of Excellence, no statistical differences were identified. Center-ofexcellence comparisons are limited by the small size of these cohorts relative to the total study population, with the margin for error therefore being increased. Though these COE credentialing programs require peripheral aspects (such as psychological counseling, nutritional support, etc) for bariatric program accreditation, it appears that the only real independent variable affecting outcome in a center-of-excellence program is the case volume. Evaluation of a single year, 2006, showed an improvement in certain outcomes in Medicarerecognized bariatric facilities, namely in postoperative shock and acute renal failure. Though this single year analysis eliminates the need to consider program designations across years, it does limit the period of study and magnifies potential errors. We contend our decision to 
extrapolate ACS and ASMBS COE designations across the entire study period likely affects the results little, as similar support and surgical experience both before and after the credentialing period are probably present. When the non-gastric band cohort was analyzed independently so as to definitively negate the aforementioned effect of missing outpatient gastric band data, all reported outcomes were unchanged except that the improvement in "any complication" rate of ACS-designated COE achieved statistical significance. The odds ratio was nearly unchanged.

It has previously been reported that fellowship training programs remove the "learning curve" period of skills acquisition for bariatric surgery ${ }^{16}$, while not increasing "any perioperative complications" 17 , but studies in this area are small and limited to single institutional cohorts. In our study of the actual situation present in the wider US surgical community, these training programs have now been confirmed to be safe for patients, with no difference in total complications or death rates, and a very large and strongly significant improvement in rates of intraoperative splenectomy during the bariatric procedures and of postoperative bacterial pneumonia rates. With the majority of bariatric operations now being performed through laparoscopic access methods, it is possible that advanced laparoscopic skills among fellows and their mentors improves these specific technical and postoperative outcomes. Decreased splenectomy rates were also noted to be independently associated with the presence of an accredited surgical residency program. It should be clearly noted that the performance of a bariatric operation in a hospital offering Fellowship Council-approved fellowship training does not guarantee that the fellow performed or was even a participant in the procedure; this limitation of the dataset will necessitate caution in the interpretation of associations between Fellowship training per se and outcomes, but will not nullify the association between the parent institution and the outcomes under consideration.

Examination of the influence on outcomes of surgical residency programs was concerning. Some major complications were significantly increased in this group; acute pulmonary embolism, acute myocardial infarction and other cardiac complications. Respiratory complications were lower in this subgroup, as were total complications. The cause for these observations is unknown. Sites of surgical training are usually sites of training for other specialties; perhaps the concurrent involvement of more junior trainees from other specialties is a confounder which results in higher perioperative medical complications such as these. Regardless, the findings warrant both concern and a need for urgent further investigation, preferably in a prospective and controlled manner. Small published series have previously reported no difference in complications in operations with resident participation as compared to those performed by an attending alone ${ }^{18} 19$; the present NIS-derived series, much larger and inclusive of more complications, shows that safety may indeed be compromised in teaching hospitals with surgical residency programs, though the cause of this is not able to be determined from the available NIS data.

Limitations exist in searches of these administrative databases. The major problem is identification of the procedures of interest, in this situation, bariatric operations. These datasets use codes from the International Classification of Disease, Ninth-Revision, Clinical Modification (ICD-9-CM). However, codes do not always exist for all procedures of interest. This is especially true early in the acceptance of a procedure. For example, though the first laparoscopic adjustable gastric band was placed in the U.S. in 2002, an ICD-9 code was not established until October 2004. Thus, a search of any ICD-9-based administrative dataset prior to this date would not have detected the band placement. The alteration and addition of ICD-9$\mathrm{CM}$ codes during a given calendar year further complicates analysis of newer procedures. A previous publication by the authors highlights the difficulty in accurately determining case volume of bariatric operations ${ }^{9}$. While the search strategy utilized herein has been previously widely used by many investigators, the potential problem of specificity of the codes still arises. 
Length of stay was considered by the authors as a variable likely to be dependent on the number and severity of complications - the more complications, the longer the stay - and so was not used as a variable in the modeling. Nonetheless, modeling was performed with the assumption that length of stay was independent; this showed minimal differences to the results above, and has not been included in the interests of brevity. Other studies have used total costs as a surrogate for complications ${ }^{15}$, but this is heavily dependent on individual hospital variation, and would penalize institutions providing more expensive, but not inferior care.

A further limitation of the NIS is related to the accuracy of data entry by hospital medical coders. The accuracy of coding has previously been reported as suboptimal ${ }^{20}$, though the detection of the presence of a particular diagnosis (as performed in this study) has been validated ${ }^{21}$. It is conceivable the programs with an active surgical residency might have better entry into the medical record of complications, adversely affecting the outcomes of this group. Furthermore, even if coding is correct, once a patient is discharged from hospital, that patient is essentially lost to follow-up. Readmissions are not captured.

Due to the statistical sampling methods used by the NIS, not all states and not all institutions are represented in the dataset, a problem encountered with most large administrative databases. However, a major advantage of the NIS is the completeness of information from individual reporting hospitals, which facilitates volume-outcome analysis ${ }^{15}$.

\section{Conclusions}

The hypothesized positive volume-outcome relationship of bariatric surgery has been shown with analysis of case volume as a continuous variable and without arbitrarily categorizing hospitals to case volume groups. The concept of volume-dependent center-of-excellence designation has been supported, though there is minimal association between outcomes and the credentialing process itself. Institutions offering fellowship training in the field have also been shown to be safe and to have association with improved outcomes in certain areas. The current paradigm of assuming improved outcomes as a result of the above parameters is supported.

\section{Acknowledgments}

This paper was supported in part by NIH Grants DK 034987 and DK 056350.

\section{References}

1. Ogden CL, Fryar CD, Carroll MD, Flegal KM. Mean body weight, height, and body mass index, United States 1960-2002. Adv Data 2004;347:1-17. [PubMed: 15544194]

2. Flegal KM, Carroll MD, Ogden CL, Johnson CL. Prevalence and trends in obesity among US adults, 1999-2000. JAMA 2002 Oct 9;288(14):1723-1727. [PubMed: 12365955]

3. Mizuno T, Shu IW, Makimura H, Mobbs C. Obesity over the life course. Sci Aging Knowledge Environ 2004 Jun 16;2004(24):re4. [PubMed: 15201431]

4. Smith CD. President's message. The Fellowship Council Newsletter 2007 Fall;:1-2.

5. SAGES guideline for clinical application of laparoscopic bariatric surgery. Surg Obes Relat Dis 2009 May-Jun;5(3):387-405. [PubMed: 19460678]

6. Changes in NIS Sampling and Weighting Strategy for 1998. Rockville, MD: Agency for Healthcare Research and Quality;

http://www.hcup-us.ahrq.gov/db/nation/nis/reports/Changes_in_NIS_Design_1998.pdf.

7. Santry HP, Gillen DL, Lauderdale DS. Trends in bariatric surgical procedures. JAMA 2005 Oct 19;294 (15):1909-1917. [PubMed: 16234497] 
8. Schilling PL, Davis MM, Albanese CT, Dutta S, Morton J. National trends in adolescent bariatric surgical procedures and implications for surgical centers of excellence. J Am Coll Surg 2008 Jan;206 (1):1-12. [PubMed: 18155562]

9. Kohn GP, Galanko JA, Overby DW, Farrell TM. Recent trends in bariatric surgery case volume in the United States. Surgery 2009 Aug;146(2):375-380. [PubMed: 19628098]

10. Deyo RA, Cherkin DC, Ciol MA. Adapting a clinical comorbidity index for use with ICD-9-CM administrative databases. J Clin Epidemiol 1992 Jun;45(6):613-619. [PubMed: 1607900]

11. Farrell TM, Haggerty SP, Overby DW, Kohn GP, Richardson WS, Fanelli RD. Clinical application of laparoscopic bariatric surgery: an evidence-based review. Surg Endosc 2009 May;23(5):930-949. [PubMed: 19125308]

12. Nguyen NT, Paya M, Stevens CM, Mavandadi S, Zainabadi K, Wilson SE. The relationship between hospital volume and outcome in bariatric surgery at academic medical centers. Ann Surg 2004 Oct; 240(4):586-593. discussion 593-584. [PubMed: 15383786]

13. Shikora SA, Kim JJ, Tarnoff ME, Raskin E, Shore R. Laparoscopic Roux-en-Y gastric bypass: results and learning curve of a high-volume academic program. Arch Surg 2005 Apr;140(4):362-367. [PubMed: 15837887]

14. Weller WE, Hannan EL. Relationship between provider volume and postoperative complications for bariatric procedures in New York State. J Am Coll Surg 2006 May;202(5):753-761. [PubMed: 16648015]

15. Livingston EH. Bariatric surgery outcomes at designated centers of excellence vs nondesignated programs. Arch Surg 2009 Apr;144(4):319-325. discussion 325. [PubMed: 19380644]

16. Kothari SN, Boyd WC, Larson CA, Gustafson HL, Lambert PJ, Mathiason MA. Training of a minimally invasive bariatric surgeon: are laparoscopic fellowships the answer? Obes Surg 2005 Mar; 15(3):323-329. [PubMed: 15832432]

17. Gonzalez R, Nelson LG, Murr MM. Does establishing a bariatric surgery fellowship training program influence operative outcomes? Surg Endosc 2007 Jan;21(1):109-114. [PubMed: 16960670]

18. Hwang CS, Pagano CR, Wichterman KA, Dunnington GL, Alfrey EJ. Resident versus no resident: a single institutional study on operative complications, mortality, and cost. Surgery 2008 Aug;144 (2):339-344. [PubMed: 18656644]

19. Robinson RP. The impact of resident teaching on total hip arthroplasty. Clin Orthop Relat Res 2007 Dec;465:196-201. [PubMed: 18090473]

20. Quan H, Li B, Saunders LD, et al. Assessing validity of ICD-9-CM and ICD-10 administrative data in recording clinical conditions in a unique dually coded database. Health Serv Res 2008 Aug;43(4): 1424-1441. [PubMed: 18756617]

21. Lacasse Y, Montori VM, Maltais F. Administrative database: validity of recording vs. validity of diagnosis. J Clin Epidemiol 2006 Jan;59(1):104. author reply 104-105. [PubMed: 16360570] 


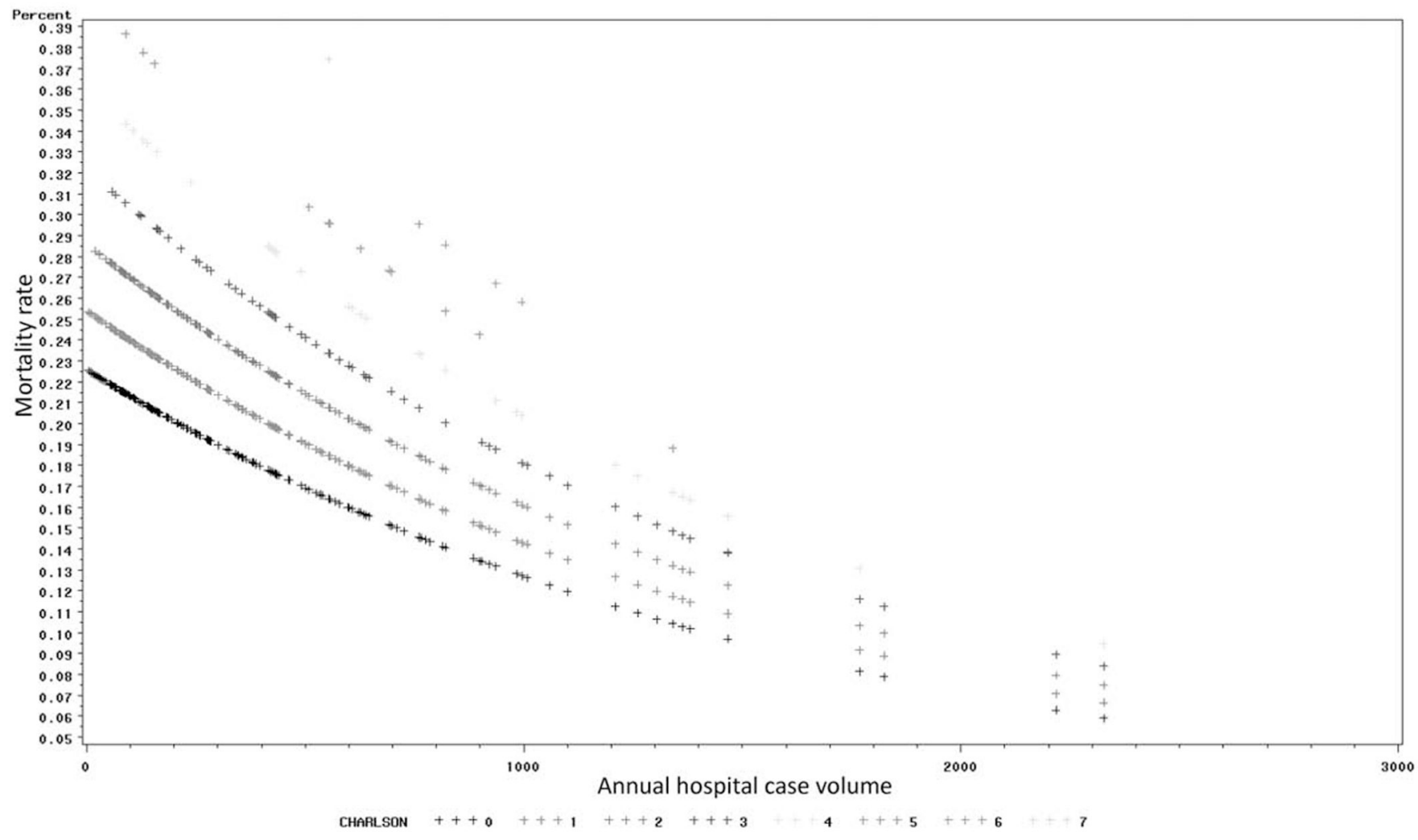

Figure 1.

Predicted hospital mortality rates in 2006, controlled for annual case volume, by Charlson comorbidity score 
Table 1

ICD 9 CM Procedure Codes Used in the Search Strategy

\begin{tabular}{|c|l|}
\hline 1 & DRG of 288 \\
\hline 2 & At least one of the following ICD-9-CM diagnosis codes: 2780, 27800, 27801, 27802 \\
\hline 3 & $\begin{array}{l}\text { Absence of any of the following diagnoses: (gastrointestinal tract neoplasms) } 1500 \text { to } \\
1599 ; \text { (inflammatory bowel disease) 5550-5569; (noninfectious colitis) 5589 }\end{array}$ \\
\hline 4 & Elective admission \\
\hline 5 & $\begin{array}{l}\text { At least one of the following ICD-9-CM procedure codes: } 435,436,437,4389,4431, \\
4438,4439,445,4468,4469,4493,4494,4495,4496,4497,4499,4550,4551,4590,4591\end{array}$ \\
\hline
\end{tabular}




\section{Table 2}

Bariatric Surgery Operation Groups

\begin{tabular}{|l|l|}
\hline Gastroplasty & 4469 \\
\hline Gastric bypass & $4431,4438,4439$ \\
\hline Malabsorptive & $4550,4551,4590$, \\
4591
\end{tabular}


Table 3

Number of Bariatric Cases (Unweighted) Performed, by Teaching Status and Bed Size of Hospitals

\begin{tabular}{|l|c|c|c|}
\hline Hospitals & $\mathbf{1 9 9 8}$ & $\mathbf{2 0 0 2}$ & $\mathbf{2 0 0 6}$ \\
\hline Teaching status, $\mathrm{n}(\%)$ & & & \\
\hline Teaching & $939(62.4 \%)$ & $7,426(60.9 \%)$ & $9,151(61.7 \%)$ \\
\hline Nonteaching & $566(37.6 \%)$ & $4,776(39.1 \%)$ & $5,685(38.3 \%)$ \\
\hline Bed size, $\mathrm{n}(\%)$ & & & \\
\hline Smallest third & $304(20.2 \%)$ & $1,196(9.8 \%)$ & $1,920(12.9)$ \\
\hline Medium third & $410(27.2 \%)$ & $2,804(23.0 \%)$ & $3,717(25.1 \%)$ \\
\hline Large third & $791(52.6 \%)$ & $8,202(67.2 \%)$ & $9,199(62.0 \%)$ \\
\hline Total bariatric cases & 1,505 & 12,202 & 14,836 \\
\hline
\end{tabular}




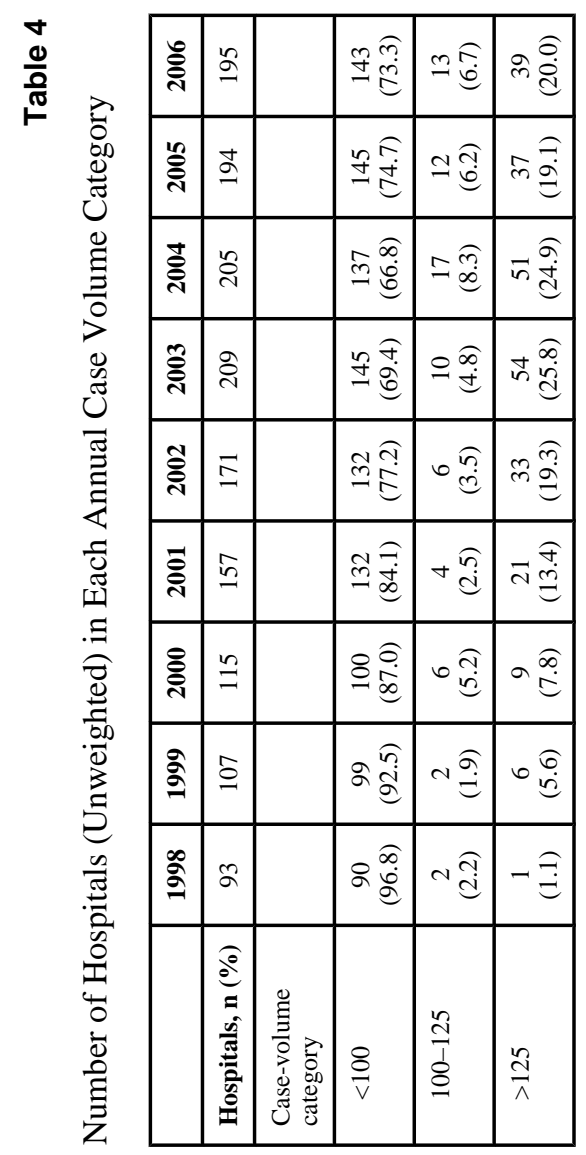

$J$ Am Coll Surg. Author manuscript; available in PMC 2011 June 1. 


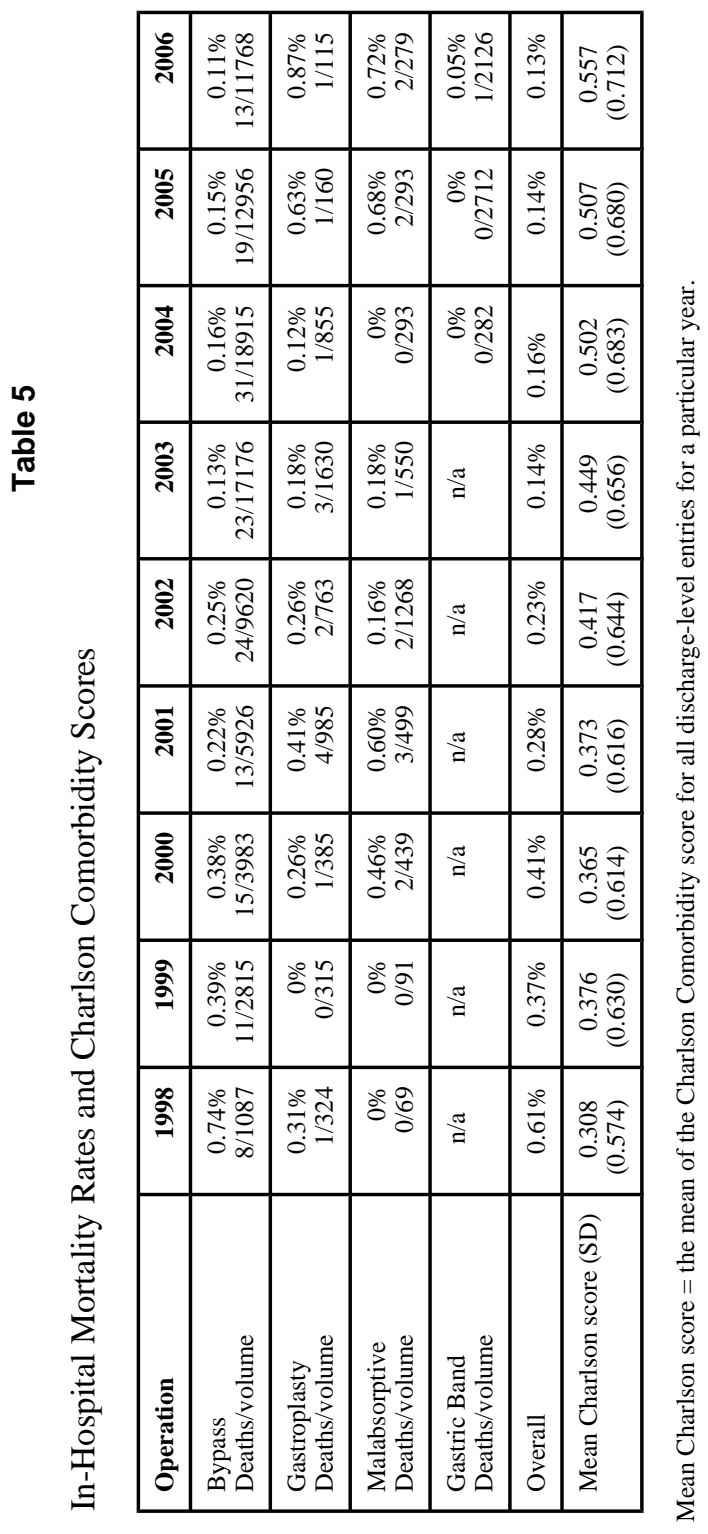

$J$ Am Coll Surg. Author manuscript; available in PMC 2011 June 1. 


\section{Table 6}

The Incremental Association of Each Bariatric Case with Annual Outcomes, Controlling for Year and for Charlson Comorbidity Scores

\begin{tabular}{|l|c|c|c|c|}
\hline & \multicolumn{2}{|c|}{ All bariatric cases } & \multicolumn{2}{c|}{ LAGB cases excluded } \\
\hline Outcome variable & Odds Ratio [95\% CI] & p Value & Odds Ratio [95\% CI] & p Value \\
\hline Any complication & $0.99937[0.99888,0.99987]$ & 0.01 & $0.99967[0.99913,1.00020]$ & 0.22 \\
\hline Death & $0.99717[0.99579,0.99856]$ & $<.0001$ & $0.99421[0.99144,0.99698]$ & $<.0001$ \\
\hline Abdominal drainage & $0.99869[0.99752,0.99985]$ & 0.03 & $0.99853[0.99727,0.99978]$ & 0.02 \\
\hline Acute DVT & $0.99989[0.99859,1.00120]$ & 0.87 & $1.00004[0.99872,1.00135]$ & 0.96 \\
\hline Acute PE & $0.99782[0.99665,0.99898]$ & 0.0002 & $0.99787[0.99669,0.99905]$ & 0.0004 \\
\hline Myocardial infarction & $0.99958[0.99832,1.0008]$ & 0.52 & $0.99968[0.99837,1.0010]$ & 0.63 \\
\hline Cardiac complications & $0.99873[0.99782,0.99964]$ & 0.01 & $0.99887[0.99804,0.99969]$ & 0.01 \\
\hline Post-op shock & $0.99776[0.99591,0.99962]$ & 0.02 & $0.99799[0.99611,0.99987]$ & 0.04 \\
\hline Splenectomy & $0.99813[0.99682,0.99943]$ & 0.01 & $0.99824[0.99694,0.99955]$ & 0.01 \\
\hline Acute renal failure & $0.99851[0.99778,0.99923]$ & $<.0001$ & $0.99854[0.99778,0.99930]$ & 0.0002 \\
\hline Acute CVA & $1.00038[0.99878,1.00199]$ & 0.64 & $1.00058[0.99888,1.00229]$ & 0.50 \\
\hline Bacterial pneumonia & $0.99738[0.99629,0.99848]$ & $<.0001$ & $0.99747[0.99640,0.99855]$ & $<.0001$ \\
\hline Respiratory failure & $0.99896[0.99806,0.99986]$ & 0.02 & $0.99900[0.99810,0.99990]$ & 0.03 \\
\hline
\end{tabular}

$\mathrm{CI}=$ confidence interval; DVT $=$ deep vein thrombosis, $\mathrm{PE}=$ pulmonary embolus, $\mathrm{CVA}=$ cerebrovascular accident.

LAGB = laparoscopic adjustable gastric band. 
Table 7

The Association of a Fellowship Program with Bariatric Outcomes, Controlling for Year, Charlson Comorbidity Scores and Case Volume

\begin{tabular}{|l|r|c|c|c|}
\hline & \multicolumn{2}{|c|}{ All bariatric cases } & \multicolumn{2}{c|}{ LAGB cases excluded } \\
\hline Outcome variable & Odds Ratio [95\% CI] & p Value & Odds Ratio [95\% CI] & p Value \\
\hline Any complication & $1.03358[0.84317,1.26699]$ & 0.75 & $0.99922[0.80932,1.23369]$ & 0.99 \\
\hline Death & $0.66476[0.35494,1.24500]$ & 0.20 & $0.69033[0.38916,1.22457]$ & 0.21 \\
\hline Abdominal drainage & $0.87260[0.46393,1.6413]$ & 0.67 & $0.87634[0.46545,1.6499]$ & 0.68 \\
\hline Acute DVT & $1.91700[1.16064,3.1663]$ & 0.01 & $1.86921[1.12680,3.1008]$ & 0.02 \\
\hline Acute PE & $1.58658[0.99020,2.54214]$ & 0.06 & $1.55527[0.96399,2.50922]$ & 0.07 \\
\hline Myocardial infarction & $1.24244[0.65816,2.34543]$ & 0.50 & $1.24444[0.66039,2.34502]$ & 0.50 \\
\hline Cardiac complications & $1.07474[0.82888,1.39351]$ & 0.59 & $1.03638[0.79801,1.34595]$ & 0.79 \\
\hline Post-op shock & $1.12195[0.57845,2.17608]$ & 0.73 & $1.07552[0.55178,2.09636]$ & 0.83 \\
\hline Splenectomy & $0.2853[0.14351,0.5672]$ & 0.0003 & $0.27520[0.13690,0.5533]$ & 0.0003 \\
\hline Acute renal failure & $1.03222[0.71208,1.49628]$ & 0.87 & $1.01519[0.69806,1.47642]$ & 0.94 \\
\hline Acute CVA & $1.57928[0.76980,3.2400]$ & 0.21 & $1.53242[0.74058,3.1709]$ & 0.25 \\
\hline Bacterial pneumonia & $0.65898[0.46566,0.93254]$ & 0.02 & $0.64864[0.45812,0.91839]$ & 0.01 \\
\hline Respiratory failure & $0.76626[0.49246,1.19229]$ & 0.24 & $0.75973[0.48679,1.18571]$ & 0.23 \\
\hline
\end{tabular}


Table 8

The Association of a Surgical Residency with Bariatric Outcomes, Controlling for Year, Charlson Comorbidity Scores and Case Volume

\begin{tabular}{|l|c|c|c|c|}
\hline & \multicolumn{2}{|c|}{ All bariatric cases } & \multicolumn{2}{c|}{ LAGB cases excluded } \\
\hline Outcome variable & Odds Ratio [95\% CI] & p Value & Odds Ratio [95\% CI] & p Value \\
\hline Any complication & $0.98162[0.84994,1.13370]$ & 0.80 & $0.95383[0.82519,1.10251]$ & 0.52 \\
\hline Death & $1.06404[0.77196,1.46662]$ & 0.70 & $1.06901[0.78008,1.46495]$ & 0.68 \\
\hline Abdominal drainage & $1.29390[0.85857,1.94996]$ & 0.22 & $1.29257[0.85821,1.9467]$ & 0.22 \\
\hline Acute DVT & $1.49852[0.99341,2.2604]$ & 0.05 & $1.47783[0.97945,2.2298]$ & 0.06 \\
\hline Acute PE & $1.89062[1.33460,2.67829]$ & 0.0003 & $1.87812[1.31897,2.67431]$ & 0.0005 \\
\hline Myocardial infarction & $1.70417[1.09085,2.66233]$ & 0.02 & $1.72402[1.10156,2.69823]$ & 0.02 \\
\hline Cardiac complications & $1.43617[1.13825,1.81207]$ & 0.002 & $1.40677[1.11948,1.76777]$ & 0.003 \\
\hline Post-op shock & $1.22331[0.73227,2.04363]$ & 0.44 & $1.18983[0.71210,1.98807]$ & 0.51 \\
\hline Splenectomy & $0.5928[0.38231,0.9192]$ & 0.02 & $0.5842[0.37705,0.9052]$ & 0.02 \\
\hline Acute renal failure & $0.95284[0.75905,1.19612]$ & 0.68 & $0.94075[0.74727,1.18433]$ & 0.60 \\
\hline Acute CVA & $1.37825[0.75522,2.5152]$ & 0.30 & $1.35308[0.74370,2.4618]$ & 0.32 \\
\hline Bacterial pneumonia & $0.61414[0.47645,0.79162]$ & 0.0002 & $0.60970[0.47322,0.78554]$ & 0.0001 \\
\hline Respiratory failure & $0.61766[0.47520,0.80283]$ & 0.0003 & $0.61420[0.47221,0.79889]$ & 0.0003 \\
\hline
\end{tabular}


己

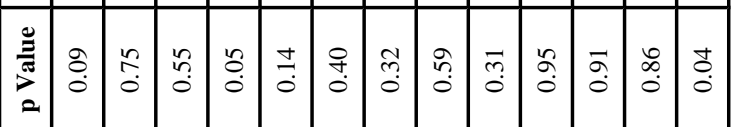

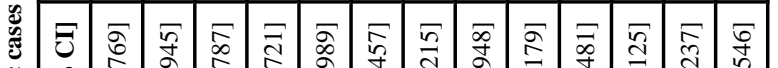

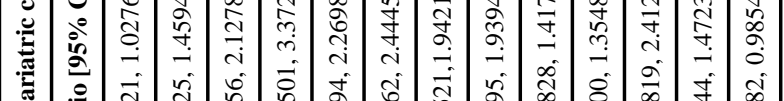

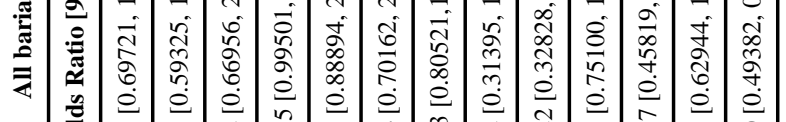

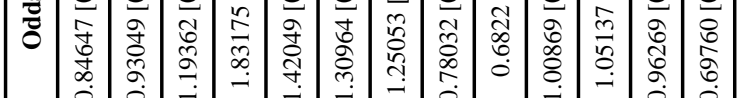

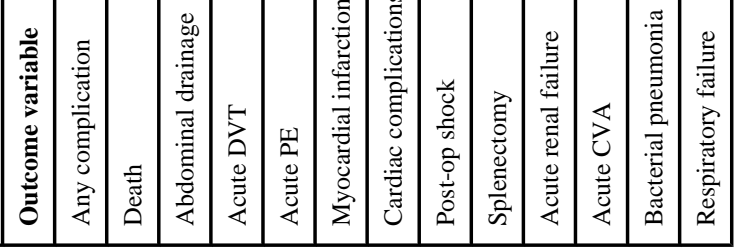




\section{Table 10}

The Association of Medicare Recognition as a Bariatric Facility in 2006 with Bariatric Surgery Outcomes, Controlling for Charlson Comorbidity Scores and Case Volume

\begin{tabular}{|c|c|c|c|c|}
\hline \multirow[b]{3}{*}{ Outcome variable } & \multicolumn{4}{|c|}{ Medicare COE 2006} \\
\hline & \multicolumn{2}{|l|}{ All bariatric cases } & \multicolumn{2}{|c|}{ LAGB cases excluded } \\
\hline & Odds Ratio [95\% CI] & p Value & Odds Ratio [95\% CI] & p Value \\
\hline Any complication & $0.88787[0.65239,1.20836]$ & 0.45 & $0.86515[0.64140,1.16695]$ & 0.34 \\
\hline Death & $0.41868[0.11205,1.56439]$ & 0.20 & $0.39578[0.10752,1.45683]$ & 0.16 \\
\hline Abdominal drainage & $0.75848[0.23948,2.40223]$ & 0.64 & $0.79142[0.24639,2.54205]$ & 0.69 \\
\hline Acute DVT & $0.82469[0.20220,3.36360]$ & 0.79 & $0.79570[0.18668,3.39147]$ & 0.76 \\
\hline Acute PE & $0.67901[0.17310,2.66357]$ & 0.58 & $0.62743[0.18060,2.17972]$ & 0.46 \\
\hline Myocardial infarction & $0.66977[0.29404,1.52560]$ & 0.34 & $0.68378[0.27479,1.70153]$ & 0.41 \\
\hline Cardiac complications & $0.69183[0.37128,1.28914]$ & 0.25 & $0.69275[0.37684,1.27347]$ & 0.24 \\
\hline Post-op shock & $0.10069[0.01271,0.79766]$ & 0.03 & $0.08292[0.00961,0.71521]$ & 0.02 \\
\hline Splenectomy & $0.90326[0.13924,5.85946]$ & 0.92 & $0.96263[0.16831,5.50550]$ & 0.97 \\
\hline Acute renal failure & $0.55166[0.32180,0.94570]$ & 0.03 & $0.50919[0.29815,0.86964]$ & 0.01 \\
\hline Acute CVA & $0.16782[0.01937,1.45356]$ & 0.11 & $0.18875[0.02176,1.63712]$ & 0.13 \\
\hline Bacterial pneumonia & $0.82559[0.41386,1.64694]$ & 0.59 & $0.80063[0.41432,1.54712]$ & 0.51 \\
\hline Respiratory failure & $0.65212[0.27385,1.55287]$ & 0.33 & $0.66978[0.29172,1.53776]$ & 0.34 \\
\hline
\end{tabular}

